\title{
Enmarcar, narrar y convencer: el papel de la información y las ideas en el proceso político de la UE*
}

\author{
Framing, narrating and convincing: \\ the role of information and ideas in the EU policy process \\ Luis Bouza García \\ Área de Ciencia Política, Universidad de Salamanca
}

doi: $10.18543 /$ ced-55-2016pp61-83

Sumario: I. Introducción.-II. Establecimiento de la agenda en un espacio público fragmentado.-III. Una sociología política de la creación de conocimientos sobre la UE: los usos del conocimiento europeo. 1. Una sociología de los usos de Europa. 2. El conocimiento como recurso: lobbying informacional.-IV. El conocimiento y la información como formas de influencia. 1. Diferentes tipos de coaliciones. 2. Algunas implicaciones metodológicas.-V. Conclusión.-VI. Bibliografía.

Resumen: Desde mediados de los años 90 los análisis sobre políticas públicas están inmersas en el llamado «giro argumentativo». Estos enfoques desarrollados en EEUU llegaron sin embargo rápidamente al estudio del proceso político comunitario. Esto resulta llamativo debido a que este es el periodo de vigencia de la teoría de la decisión racional en los estudios sobre la UE y a que ésta carece de un espacio público. Sin embargo argumentamos que este giro argumentativo se puede entender debido a algunas similitudes en el proceso político puesto que la UE es también un sistema de pesos y contrapesos caracterizado por la intervención de múltiples actores en el cual la alineación de marcos interpretativos en procesos de medio y largo plazo contribuye a la creación de las coaliciones amplias que se necesitan para el establecimiento de la agenda y la transformación de políticas públicas en este tipo de sistemas. Este artículo repasa el uso enfoques epistémicos o centrados en las ideas para explicar el proceso político europeo. Desde el punto de vista teórico se señala la utilidad de analizarlos para estudiar la agenda desde una perspectiva de actores amplia y como mecanismo heurístico que permite estudiar la agencia de distintos intervinientes en los procesos de establecimiento de la agenda en la correspondencia entre estrategias de acción colectiva. Estos enfoques también permiten evaluar hipótesis relativas a la importancia del aprendizaje y la alineación de marcos cognitivos. Desde el punto de vista epistemológico y metodológico se señalan

* Recibido el 19 de mayo de 2016, aceptado el 21 de junio de 2016. 
las ambigüedades pero también potencialidades para utilizar gran cantidad de datos y combinar procedimientos cuantitativos y cualitativos.

Palabras clave: Proceso político europeo, establecimiento de la agenda, marcos interpretativos, coaliciones, grupos de interés.

Abstract: Since the mid-90s public policy analyses has experienced the socalled "argumentative turn». These approaches developed in the US but quickly became usual in the study of the EU policy process. This is striking given the dominance of the rational choice theory in EU studies during this time and because the EU lacks a public space. However the article argues that this turn can be understood thanks to some similarities in the policy process: the EU is also a system of checks and balances characterised by the involvement of diverse actors in which the alignment of interpretative frame in the medium and long term contributes to create the broad coalitions that are required to establish and change the agenda. The article reviews the contribution of epistemic or ideas-centred approaches in explaining the European policy process. From the theoretical point of view these analyses provide a wide perspective on the actors involved and provide heuristic mechanisms to analyse actors' agency in the correspondence between collective action strategies and the institutional agendas. These approaches also formulate hypotheses on the importance of learning and interpretive frame alignment that imply some epistemological and methodological ambiguities but also shed potential for large $N$ and mixed methods designs.

Keywords: European policy process, agenda-setting, interpretive frames, coalitions, interest groups.

\section{Introducción}

El día 23 de abril de 2013 - Día Internacional del Libro en honor de los grandes creadores de relatos que fueron Shakespeare y Cervantes - el entonces presidente de la Comisión Europea José Manuel Durao Barroso expuso la idea de que la Unión necesita un nuevo relato, puesto que el creciente descontento y desconcierto de los ciudadanos ante el rumbo de la UE confirmaría que la justificación original de la integración europea en torno a la pacificación del continente se estaría agotando debido a su propio éxito. La argumentación de Barroso es interesante en más de un aspecto, pero en el contexto de nuestra contribución a este número especial nos lleva a interrogarnos sobre las explicaciones del proceso político de la UE como un régimen de producción de conocimientos, explicaciones y creencias. En la afirmación de Barroso hay por lo menos dos aspectos controvertidos: que el proyecto de integración europea desde los años 1950 hasta los 2000 se había apoyado en un relato sobre la reconciliación - lo cual contrasta con ex-

Cuadernos Europeos de Deusto 
plicaciones que indican que se habría tratado más bien de en un consentimiento pasivo por parte de los ciudadanos fundamentado en la ignorancia de dicho proceso $^{1}-$ y que las actitudes negativas hacia la UE pueden explicarse y modificarse a través de la construcción de relatos - obviando factores relativos a la situación económica, la evaluación de las políticas públicas comunitarias o las percepciones de la UE por diferentes categorías de la población-.

Si bien esta argumentación es una construcción interesada no deja de resultar ilustrativa del interés de los estudiosos de la UE y de las propias instituciones europeas por explicaciones centradas en el conocimiento, la argumentación y la creación de símbolos y relatos. Este énfasis en dicho enfoque resulta especialmente llamativo ante la conocida ausencia de un espacio público europeo, la debilidad de los actores que deberían realizar la mediación cultural de la política europea - medios e intelectuales ${ }^{2}-$ y las diferencias en la estructura de la opinión pública hacia la UE entre los distintos Estados miembros, todos ellos factores que sugerirían un cierto escepticismo en torno a la posibilidad de explicar la política europea mediante factores cognitivos. Este interés por las explicaciones del proceso político comunitario en función de la capacidad de los actores de convencer a los demás no es nueva puesto que desde hace más de una década se acumulan los enfoques deliberativos, epistémicos o de análisis de los marcos interpretativos.

En este artículo intentamos explicar este interés por los enfoques cualitativos a pesar de la debilidad del espacio público y de la comunicación de la UE. El artículo repasa los enfoques sobre el uso del conocimiento y la información por parte de los actores del proceso político con el argumento de que el éxito de estos modelos tiene que ver con los modos de intervención en el establecimiento de la agenda en un espacio público segmentado como el europeo así como con el tipo de actores que predomina en el mismo. El artículo toma en serio la noción de uso de los marcos al apoyarse en un modelo de acción estratégica de la sociología política de la integración europea que subraya que las ideas contribuyen por una parte a definir las preferencias de los actores $-\mathrm{y}$ limitar por lo tanto su margen de maniobra - pero que también constituyen un recurso para la acción colectiva.

La primera parte del artículo aplica dos modelos de establecimiento de la agenda - el del equilibrio puntuado y el incremental- a la relación entre el proceso político y el espacio público de producción y circulación de

${ }^{1}$ Leon Lindbergand y Stuart Scheingold, Europe's Would Be Polity (Englewood Cliffs: Prentice Halls, 1970).

2 Justine Lacroix y Kalypso Nicolaïdis, European Stories: Intellectual Debates on Europe in National Contexts (Oxford: Oxford University Press, 2010). 
relatos. En la segunda sección el artículo se propone un modelo sociológico sobre el uso estratégico del conocimiento en el proceso político. Este modelo de racionalidad limitada en el cual se entiende que los actores actúan en función de sus creencias pero que también tienen un margen para actuar estratégicamente usando el discurso como herramienta es compatible con todos los enfoques que señalan la importancia de la información y las coaliciones y redes en el proceso político comunitario. La tercera sección analiza los aspectos comunes y las diferencias entre algunos de los modelos sobre el conocimiento en el proceso político europeo - marcos interpretativos, coaliciones promotoras o comunidades epistémicas - y presenta algunas implicaciones metodológicas.

\section{Establecimiento de la agenda en un espacio público fragmentado}

Al preguntarnos por la importancia del análisis epistémico en el proceso político parece necesario interrogarse por la relación entre el sistema político comunitario y el o los espacios públicos europeos. La cuestión del espacio público europeo adquirió gran importancia en los años 2000 tanto desde estudios de teoría política que se preguntaban por la contribución de la deliberación a la democratización de la $\mathrm{UE}^{3}$ como desde enfoques de sociología política que buscaban analizar si la europeización de políticas y actores y por lo tanto la expresión de demandas hacia el sistema político se vería acompañada del desarrollo de un espacio de debate a escala europea. Estos análisis han sido menos frecuentes tras la aprobación del Tratado de Lisboa, si bien la crisis del euro parece haber contribuido más al desarrollo de debates de alcance europeo al mismo tiempo en todos los Estados miembro que los de ratificación de los tratados en los años 2000.

En todo caso las conclusiones de casi todas estas contribuciones están claras: la ausencia de un espacio público europeo no se debe a una ausencia de interés objetiva de las políticas de la UE para el público sino a la desconexión entre el proceso político comunitario y la opinión pública. Eriksen ${ }^{4}$ señala que más que la inexistencia de un espacio público europeo se debe hablar de su fragmentación. Lejos de una unanimidad sobre las políticas europeas existe un claro pluralismo de elites caracterizado por un intenso debate sobre políticas públicas europeas en los foros institucionales y es-

3 Damien Chalmers, «The reconstitution of European public spheres», European Law Journal, 9, 2 (2003): 127-189.

${ }^{4}$ Erik Odvar Eriksen, «Conceptualising European public spheres. General, segmented and strong publics», en J. E. Fossum \& P. Schlesinger, eds. The European Union and the Public Sphere. A communicative space in the making? (London: Routledge, 2007). 
pecializados. Sin embargo este debate relativamente intenso tiende a no ampliarse hacia un público más amplio, debido fundamentalmente a la escasa competencia entre los actores del espacio intermedio que estarían llamados a realizar dicha mediación. Esto se debe a que en el juego político a nivel comunitario la movilización de la opinión pública es una estrategia costosa y poco exitosa por varios motivos. En primer lugar encontramos la falta de competencia por los puestos institucionales en el sistema político ${ }^{5}$, seguida de la especialización de los grupos de interés y su dificultad de conectar a sus miembros con el sistema político europeo ${ }^{6}$ y las escasas ocasiones de rendición de cuentas ante un electorado europeo y no uno fragmentado ${ }^{7}$. Todas estas afirmaciones comparten la idea de que en las propias dinámicas de funcionamiento en el espacio público intermedio los actores carecen de incentivos para intentar asociar a sus miembros con mayor intensidad, lo cual dificulta el desarrollo de un espacio público europeo.

La literatura sobre el proceso de establecimiento de la agenda se apoya en dos modelos alternativos a la hora de explicar el cambio: el modelo del equilibrio puntuado frente al del incrementalismo ${ }^{8}$. Las diferencias entre ambos modelos se encuentran en la frecuencia e intensidad del cambio en la agenda así como en el modo del cambio. En cuanto a la primera dimensión el modelo del equilibrio puntuado señala que en la mayor parte de políticas predomina la estabilidad y la continuidad. Puesto que la capacidad de atención de los decisores es limitada lo más frecuente es que una vez que un determinado asunto alcanza un equilibrio los actores carezcan de incentivos para seguir concentrándose en el mismo. Por lo tanto las explicaciones sobre las alteraciones de dichos equilibrios tienen que ver con la incidencia de acontecimientos externos que focalizan la atención política y mediática (conocidos como «focusing events» en la literatura internacional), favoreciendo bruscos cambios de rumbo de la agenda política para responder a cambios sobrevenidos. En la UE estos acontecimientos suelen relacionarse con la tradicional argumentación de que la UE sólo avanzaría mediante las

5 Andreas Follesdal y Simon Hix.. «Why There is a Democratic Deficit in the EU: A Response to Majone and Moravcsik», Journal of Common Market Studies, 44.3 (2006): 552

${ }^{6}$ Christine Mahoney, «The Role of Interest Groups in Fostering Citizen Engagement: The Determinants of Outside Lobbying», en William Maloney y Jan van Deth (eds), Civil Society and Governance in Europe. From National to International Linkages (Cheltenham: Edward Elgar, 2008).

7 Thierry Chopin, «Towards a Genuine European Executive Power: from Governance to Government», European Issues, 274 (2013), http://www.robert-schuman.eu/doc/questions_ europe/qe-274-en.pdf.

${ }^{8}$ Frank R. Baumgartner, Bryan D. Jones y Peter B. Mortensen, «Punctuated equilibrium theory: Explaining stability and change in public policymaking», en Paul A. Sabatier y Christopher Weible (eds), Theories of the policy process (2014), 59-62. 
crisis. Algunos ejemplos recientes podrían ser la creación de mecanismos de rescate financiero a los Estados del euro - cuando hasta entonces el tratado sugería que esto era imposible - y el cierre de fronteras entre Estados europeos como respuesta a la crisis de refugiados durante el verano y el otoño de 2015, poniendo en cuestión, siquiera simbólicamente, el principio de libre circulación. Sin embargo este enfoque tiene límites a la hora de explicar el cambio en las agendas europeas ${ }^{9}$ puesto que dichos acontecimientos focalizadores son excepcionales, no sólo por su importancia sino por la dificultad de mediatizar hechos de este tipo de tal forma que se cree suficiente presión sobre las instituciones para modificar las políticas en vigor. Siguiendo la lógica de la economía de la atención, no basta con que un acontecimiento sea significativo y cuestione las preferencias de las instituciones de la UE, sino que las instituciones del sistema político tienen que sentir que sus preferencias se ponen en cuestión por parte de la opinión pública. Por lo tanto el tratamiento mediático de estos acontecimientos resulta decisivo a la hora de posibilitar que se pueda ejercer efectivamente una presión significativa para cambiar la agenda. En este contexto por lo tanto la debilidad de los medios transnacionales, la disociación entre decisiones gubernamentales en la UE y la rendición de cuentas a escala nacional, la diversidad de enfoques habituales sobre un mismo acontecimiento y lo extraordinario de que un mismo hecho interese a todos los medios de Europa al mismo tiempo hace que los cambios bruscos de agenda sean espectaculares pero raros.

La mayor parte de agendas comunitarias ${ }^{10}$ parecen poder explicarse mejor a través del incrementalismo. La idea subyacente detrás de este enfoque es que la forma habitual de cambio en la agenda no pasa por los cambios bruscos debido a acontecimientos externos noticiosos sino por la acumulación de numerosos cambios que se van superponiendo en el tiempo como respuestas contingentes a demandas separadas. Esto significa que más que una gran batalla decisiva los procesos de establecimiento de la agenda son una serie de escaramuzas constantes e interrelacionadas cuyo sentido debe interpretarse en el plano estratégico. Este enfoque además le da un mayor peso a la acción intencionada para situar asuntos en la agenda por parte de actores interesados, emprendedores de causa y redes de políticas públicas. El énfasis en el papel de los actores también le presta atención a las resistencias a los cambios en la agenda por parte de los actores que se benefician del statu quo. De esta manera este enfoque sugiere que el margen para el cambio en cada oportunidad es menor. Esto tiene dos consecuencias importantes:

9 Véase sin embargo Sebastian Princen, «Punctuated equilibrium theory and the European Union». Journal of European Public Policy, 20, 6 (2013).

${ }^{10}$ Sebastian Princen,. Agenda-setting in the European Union. (Basingstoke: Palgrave Macmillan, 2009). 
por una parte los cambios en la agenda no son tanto el resultado de oportunidades históricas («critical junctures») como de la acumulación de cambios de menor alcance. En segundo lugar además resulta menos evidente localizar los cambios de la agenda en un único foro de elaboración de políticas públicas sino que pueden ser resultado de la acumulación de cambios relativamente menos importantes en varios campos que en principio parecerían desconectados, lo que Kingdon ${ }^{11}$ conceptualizó como la confluencia de varios flujos («policy streams»).

Este enfoque es más coherente con la estructura del sistema político de la Unión Europea, que se puede caracterizar como un sistema de gobernanza en base a pesos y contrapesos más que como un sistema basado en la rendición de cuentas respecto a la capacidad de procesar rápidamente nuevas demandas o resolver nuevos problemas puesto que aunque la atención política sea limitada en un sistema caracterizado por la multiplicidad de puntos de acceso a la agenda existen numerosas ventanas de oportunidad para formular demandas que las instituciones de la UE puedan procesar. Esta concepción esta próxima a la de la literatura sobre la gobernanza multinivel de la UE: al igual que en EEUU y en algunas democracias consociativas europeas el sistema institucional de la UE se caracteriza por la multiplicidad de puntos de acceso a la elaboración de políticas, la negociación permanente entre un gran número de actores y la existencia de numerosos actores con capacidad de veto ${ }^{12}$.

En primer lugar el modo de elaboración de las políticas públicas de la UE ofrece una gran cantidad de puntos de acceso a la agenda, a pesar de que el monopolio de la iniciativa legislativa por parte de la Comisión pueda dar una impresión diferente. Esto se debe al funcionamiento de la UE como un sistema de gobernanza multinivel, en el cual está institucionalizada la representación de diferentes tipos de intereses tales como los territoriales, nacionales y comunitarios. Además las propias instituciones están especializadas en la representación funcional y política, directa e indirecta de cada uno de esos intereses. Dichas instituciones necesitan cooperar para alcanzar decisiones, ofreciendo numerosos cauces de acceso a la agenda en diferentes momentos. Por último el giro de la Comisión tras la caída de la Comisión Santer hacia formas manageriales de elaboración de políticas públicas supone una formalización de las oportunidades de acceso - a veces conocido como «stakeholderismo»- para aquellos actores afectados que tienen los recursos necesarios para participar.

11 John W. Kingdon Agendas, Alternatives, and Public Policies (New York: Longman, 2003, 2 ed.), 86-89

${ }^{12}$ George Tsebelis y Xenophon Yataganas, «Veto Players and Decision-making in the EU After Nice», Journal of Common Market Studies, 40,2 (2002): 283-307. 
En segundo lugar esta multiplicación de los puntos de acceso tiene que ver con el pluralismo del modo de intermediación de intereses en la UE. La progresiva transferencia de competencias hacia las instituciones de la UE y el activismo de determinados grupos ha dado lugar al desarrollo y consolidación de un ecosistema de grupos organizados a escala europea y con frecuencia muy especializados en la defensa de intereses sectoriales. A pesar de algunas inclinaciones hacia una organización corporatista de dicho entorno en los años 90, en lugar de designar interlocutores privilegiados las instituciones europeas han ido diseñando formas de interacción formalizadas que permitan que cualquier grupo pueda intervenir del mismo modo en la elaboración de políticas públicas, preocupándose por diseñar procedimientos para facilitar la competencia entre los mismos tal como el registro de transparencia. Este interés de las instituciones en favorecer la existencia a escala europea de múltiples grupos que se puedan contrarrestar ha ido hasta el apoyo financiero a la europeización de organizaciones ciudadanas como la Plataforma Social Europea, en lo que para unos es una estrategia neo-pluralista en la cual el sector publico apoya la diversidad pero para otros parece un caso de «captura a la inversa» en el cual las instituciones controlan, al menos parcialmente, a aquellos que intentan ejercer influencia.

En tercer lugar en la política europea existen múltiples puntos de veto tal y como puede ser la Comisión en la fase de propuestas, una coalición de varios partidos en el Parlamento o una minoría de bloqueo en el Consejo. Todo ello obliga a que los actores que intentan conseguir influencia busquen asociar a la institución más favorable a su propuesta - no todas las DG de la Comisión o Estados miembro serán igual de receptivos- y a formular las propuestas de cambio de la agenda de manera general y en el contexto de transacciones. Por lo tanto la forma más habitual de promoción de cambios en la agenda es la de las redes o coaliciones amplias que agrupan a actores de diferente tipo y nivel y que asocian tanto a actores institucionales como a activistas externos.

El resultado de todo ello es que la influencia en la agenda se ejerce a largo plazo y a través de la presencia repetida y la reputación. Todo ello tiene un efecto sobre los tipos de actores y sus estrategias de influencia. La siguiente sección analiza esta cuestión.

\section{Una sociología política de la creación de conocimientos sobre la UE: los usos del conocimiento europeo}

¿Qué tienen que ver los modos de establecimiento de la agenda con la importancia de los modelos epistémicos? El argumento central es que debido a que es más difícil usar la presión de la opinión pública para fomentar 
el cambio, este se produce a través de la convergencia de las posiciones de los diferentes actores cuyo concurso es necesario para la toma de decisiones. No todos los enfoques asumen que estas estrategias consiguen modificar las preferencias de los actores ${ }^{13}$, aunque todos aceptan que la información y el conocimiento ejercen un papel clave en la articulación de coaliciones, ya sea mediante el intercambio y las transacciones o mediante el convencimiento.

\section{Una sociología de los usos de Europa}

Desde hace aproximadamente dos décadas se desarrolla sobre todo en Francia un enfoque de sociología política que se puede entender en el contexto de la innovación teórica en torno a la integración europea ${ }^{14}$, con la intención de superar el debate entre neofuncionalismo e intergubernamentalismo, o al menos de proporcionar enfoques de alcance intermedio e intentar abrir las numerosas «cajas negras» de ambas teorías.

Estas teorías, próximas del nuevo institucionalismo sociológico en ciencia política, encuentran su dimensión sociológica en el estudio de los fundamentos sociales y los usos del poder en la integración europea. Para la mayor parte de autores se trata de analizar en qué medida las trayectorias de los actores o las decisiones de las instituciones son resultado de condicionamientos estructurales ya sean de nivel sistémico, institucional o individual. En este sentido buscan alejarse del paradigma de las teorías de la decisión sobre la capacidad de los actores de diseñar estrategias de maximización de sus intereses y señalan que los actores se mueven en entornos de racionalidad limitada, al tiempo que cambian el enfoque de los Estados o las instituciones a los actores concretos. En este sentido no conciben la Comisión Europea o el Estado como actores monolíticos sino como un conjunto de actores y servicios con intereses, formas de hacer las cosas e incluso ethos colectivos diferenciados.

Los enfoques sociológicos buscan entender las motivaciones de los actores en entornos de racionalidad limitada, por lo que los valores, ideas y en general formas de ver el mundo adquieren una importancia decisiva. Sin embargo los enfoques de sociología política también se alejan del constructivismo puesto que no entienden que las identidades, significados o la naturaleza construida del mundo social condicionen la actuación de los actores.

13 Sobre la sociología cognitiva del proceso politico, ver Pierre Muller, «L'analyse cognitive des politiques publiques: vers une sociologie politique de l'action publique», Revue française de science politique (2000), 189-207.

${ }_{14}$ Sabine Saurugger (dir), Les approches sociologiques de l'intégration européenne: Perspectives critiques. Politique européenne, 25 (2008): 5-230. 
En este sentido Woll y Jacquot ${ }^{15}$ señalan que los enfoques sociológicos conciben la relación entre estructura y agencia desde la noción de acción estratégica, en la cual los actores tienen una relación estructural y estructurante con las ideas, en la cual las mismas condicionan las posibilidades de acción y las nociones del valor de la acción social pero en la cual los actores también pueden construir y usar las nociones como mejor conviene a los objetivos que buscan. Desde este punto de vista por lo tanto el conocimiento y las ideas se pueden analizar como un recurso especialmente valioso en un proceso político caracterizado por la diversidad de actores y la necesidad de formar coaliciones en base a la reputación y la acción a largo plazo.

\section{El conocimiento como recurso: lobbying informacional}

Estos son factores que se tienen en cuenta desde hace tiempo en los análisis sobre la influencia de los grupos de interés en la UE y sobre los que empieza a haber una evidencia más sistemática. El análisis parte de la consideración del sesgo estructural de los sistemas de intermediación de intereses en el capitalismo hacia los intereses económicos ${ }^{16}$. Sin embargo la afirmación de esta predominancia estructural resulta difícil de compatibilizar con la evidencia de la activa movilización de dichos intereses: si se trata de intereses estructurales y que resultan privilegiados se podría esperar que las instituciones los tuvieran en cuenta de todos modos y por lo tanto no parece lógico que dichos grupos empleen recursos y se expongan públicamente para defenderlos. Por lo tanto los trabajos actuales sobre representación de intereses en la UE han buscado explicitar mejor por lo tanto el fenómeno de la movilización en un contexto pluralista como es la UE. En este sentido se ha señalado en primer lugar que el sistema europeo de representación de intereses es una forma de pluralismo de elite ${ }^{17}$ en el que la movilización de numerosos intereses económicos y empresariales no deja de ser una manifestación de la naturaleza competitiva del pluralismo, una concepción de la intermediación de intereses basada el elitismo que enfatiza la competencia más que la cooperación entre las elites ${ }^{18}$. De este modo la sobre-representa-

15 Sophie Jacquot y Cornelia Woll, «Action publique européenne: les acteurs stratégiques face à l'Europe», Politique européenne, 2 (2008): 161-192.

${ }^{16}$ Charles E Lindblom, Politics and markets: the world's political-economic systems (New York: Basic, 1977).

17 Reiner Eising, «The access of business interests to EU institutions: towards elite pluralism?», Journal of European Public Policy, 14, 3 (2007): 384-403.

18 Robert A. Dahl, «A Critique of the Ruling Elite Model», American Political Science Review, 52, 2, (1958): 463-464. 
ción de los intereses económicos en la UE puede tener que ver con diferencias fundamentales entre ellos. De esta manera la literatura no ha dejado de señalar la tendencia a la fragmentación de los intereses empresariales hasta el punto que por ejemplo la industria del vidrio está representada por 7 organizaciones diferentes ${ }^{19}$ mientras que las organizaciones de intereses generales han tendido a crear una gran asociación por sector, en parte por la presión institucional en este sentido ${ }^{20}$. Por otra parte se ha desarrollado recientemente un modelo de análisis sobre el intercambio de recursos: en un contexto pluralista las organizaciones necesitan intervenir para que las instituciones les escuchen a ellas y no a sus rivales, y para conseguirlo necesitan ofrecerles recursos a los que dichas instituciones no pueden acceder. En los últimos años numerosos autores han enfatizado la importancia de la información técnica para una pequeña administración generalista como es la Comisión Europea ${ }^{21}$.

La información es uno de los principales recursos intercambiados entre instituciones y grupos de interés en la UE. Aunque esta motivación también está presente en intercambios informales, hasta cierto punto podríamos decir que es la importancia de este recurso la que ha determinado la creciente formalización de los intercambios con los grupos de interés. Ya en su primera comunicación sobre los intercambios con los grupos de interés la Comisión (1992) subraya su papel como proveedores de información. La década en torno del fallido Tratado Constitucional rechazado en 2005 dio lugar a una serie de mecanismos participativos de poco recorrido en relación a su capacidad de asociar a los ciudadanos ${ }^{22}$, si bien en otro plano los diez años entre el libro blanco sobre la gobernanza de la UE de 2001 y la revisión del registro de transparencia (2011) han dado lugar a intercambios cada vez más reglados entre Comisión y grupos de interés ${ }^{23}$. En dicho proceso se ha ido desplazando el foco del debate desde la selección de las organizaciones que podían reunirse con la Comisión en base a criterios de re-

19 Justin Greenwood, Interest representation in the European Union (London: Palgrave Macmillan, 2011), 15-16; 92-99.

${ }^{20}$ Greenwood, Interest ... 130-137

${ }^{21}$ Peter Bouwen. y Margaret McCown, «Lobbying versus litigation: political and legal strategies of interest representation in the European Union», Journal of European Public Policy 14: 2007; Adam W. Chalmers, «Interests, influence and information: comparing the influence of interest groups in the European Union», Journal of European Integration 33 (2011); Heike Klüver, Lobbying in the European Union: interest groups, lobbying coalitions, and policy change. (Oxford: Oxford University Press, 2013).

${ }_{22}$ Luis Bouza García, Participatory Democracy and Civil Society in the EU: AgendaSetting and Institutionalisation. (2015), 130-143.

${ }^{23}$ Stijn Smismans, «European civil society: Shaped by discourses and institutional interests», European law journal 9.4 (2003): pp. 473-475. 
presentatividad hacia el intercambio de información. De esta manera cada vez son menos frecuentes las llamadas consultas dirigidas con un grupo selecto de organizaciones mientras que es más frecuente encontrar consultas online abiertas a cualquier organización ${ }^{24}$. Lo más significativo es que dichas webs de consulta se han diseñado en torno a un cuestionario en el cual la Comisión busca recabar información sobre las posiciones de los grupos, al tiempo que facilita que las organizaciones envíen información adicional. La consolidación del registro de transparencia a partir de 2011 mediante la fusión de los registros de la Comisión y el Parlamento también confirma la importancia de la gestión de la información en los intercambios con la sociedad civil, en la medida en que además de suministrar información sobre su estructura, los intereses a los que representa o los recursos que emplea, por primera vez se establece un código de buena conducta que entre otras cosas compromete a los grupos de interés a suministrar información relevante y veraz. Independientemente de la ausencia de obligaciones legales derivada de la debilidad del registro esta evolución demuestra la importancia que está adquiriendo la información en los intercambios entre Comisión y grupos de interés así como en la reputación de las organizaciones.

Además la investigación sobre grupos de interés en la UE enfatiza la importancia del lobbying en coalición. A pesar de algunos relatos sobre el éxito de un único grupo en una gran decisión - como los industrialistas europeos (ERT) en las decisiones sobre el mercado único o del Lobby Europeo de Mujeres (EWL) en la inclusión de las cláusulas sobre igualdad de género en el tratado de Ámsterdam, la capacidad de un único actor - grupo de interés, empresa, región o Estado - de promover un cambio en la agenda en solitario es muy limitado. En general la promoción de cambios en la agenda es el resultado de la confluencia de varias organizaciones en torno a una o más coaliciones cuya influencia se ejerce en el largo plazo.

Estas coaliciones son relevantes para las instituciones en varios planos. En primer lugar resuelven problemas de acción colectiva en la medida en que simplifican un panorama caracterizado por una gran diversidad de actores, de tal modo que las instituciones pueden interactuar con unos pocos interlocutores. En segundo lugar estas coaliciones son señales para la Comisión sobre el grado de apoyo a una propuesta. Aunque Mahoney 25 argumenta que las coaliciones en la UE están más motivadas por el intercambio de recursos que por la construcción de causas hacia la opinión pública, esta dimensión sigue siendo significativa porque indica el grado de resistencia en la

${ }^{24}$ Christine Quittkat, «The European Commission's online consultations: a success story?», Journal of Common Market Studies, 49,3 (2011).

${ }_{25}$ Christine Mahoney, «Networking Vs. Allying: The Decision of Interest Groups to Join Coalitions in the US and the EU», Journal of European Public Policy 14, 3 (2007) 
fase de decisión e implementación. Por último y de forma especialmente significativa las coaliciones sirven para la acumulación de recursos de diferente naturaleza. Tal y como han señalado los enfoques sociológicos sobre el proceso político comunitario los recursos no son sólo una disposición objetiva ${ }^{26}$ que permita predecir cuáles serán las coaliciones ganadoras - por ejemplo, sabemos que los grupos de interés económico obtienen menos influencia de sus recursos que las organizaciones que defienden intereses ciudadanos ${ }^{27}-$ sino que se generan en la propia interacción entre los actores y que además tienen que ser movilizados conscientemente - no basta con ser un actor objetivamente importante, es necesario estar implicado en el proceso para obtener influencia - y adaptados al proceso político, por ejemplo una propuesta de alta complejidad técnica es valiosa para las instituciones - cuyos representantes se suelen enfrentar a un «dilema del folio en blanco» regulatoriosólo en la medida en que además esté formulada en términos europeos tanto en el plano del contenido como en el lenguaje jurídico-institucional ${ }^{28}$.

A continuación abordamos en mayor detalle la cuestión de la información y la influencia. En segundo lugar nos interesamos por el papel del conocimiento y de las percepciones en la formación de diferentes tipos de coaliciones.

\section{IV . El conocimiento y la información como formas de influencia}

Resulta necesario abordar en primer lugar la cuestión de los marcos interpretativos y su aplicación al estudio del proceso político de la Unión Europea, no sólo porque son un enfoque aplicable a todos los actores - el interés por los marcos se origina en los estudios sobre comunicación ${ }^{29}$ y movimientos sociales, pero es aplicable a las estrategias de los grupos de interés ${ }^{30}$ y de las instituciones - sino porque se vincula con el modelo teórico sobre la sociología del uso del conocimiento en el proceso político.

26 Jacquot y Woll, «Action publique européenne...

27 Andreas Dür, Peter Bernhagen, y David Marshall, «Interest Group Success in the European Union When (and Why) Does Business Lose?», Comparative Political Studies, 48, 8 (2015).

${ }^{28}$ Philippe Aldrin, «Producteurs, courtiers et experts de l'information européenne», en D. Georgakakis, Le champ de l'eurocratie, (Economica, 2012)

29 Juan Diez Medrano y Emily Gray, «Framing the European Union in National Public Spheres», en Ruud Koopmans y Paul Statham, (eds), The making of a European public sphere: Media discourse and political contention (Cambridge: Cambridge University Press, 2010).

${ }^{30}$ Frida Boräng, Rainer Eising, Heike Klüver, Christine Mahoney; Daniel Naurin, Daniel Rasch y Patricja Rozbicka, P., «Identifying frames: A comparison of research methods», In- 
Los marcos interpretativos no son una ideología cerrada sino una serie de encuadres explicativos sobre el mundo político y social - que Goffman ${ }^{31}$ define como el esquema que utilizan los individuos y grupos para organizar y explicar sus experiencias sociales - que los grupos sociales compiten por imponer en la definición de la agenda política. Se trata por lo tanto de un enfoque «que toma las ideas en serio» ${ }^{32}$, pero que es necesario diferenciar en el plano epistemológico y teórico de los enfoques constructivistas. En un contexto de racionalidad limitada los actores están condicionados por sus percepciones y valores pero tienen un grado de «habilidad social» ${ }^{33}$ que les permite adaptar sus encuadres de los fenómenos sociales en función de las circunstancias o de las estrategias que estén buscando poner en marcha. Este argumento ha sido bien ilustrado por los análisis de los movimientos sociales al señalar que los marcos interpretativos son por una parte un elemento constitutivo de la identidad del propio movimiento y por otra un elemento central de sus formas de acción colectiva ${ }^{34}$.

El estudio de los marcos de referencia es un aspecto central del análisis del proceso político ${ }^{35}$, en la medida en que el proceso de establecimiento de la agenda no consiste sólo en la determinación de los temas que captan la atención sino los modos en que lo hacen. Los análisis centrados en los actores señalan que la adaptación del marco de interpretación en función de la posición de los actores en el proceso político es algo habitual. En este sentido algunos estudios sobre el funcionamiento de los foros de consultas de la Comisión Europea mencionados más arriba señalan que los grupos interesados tienden a reproducir los marcos de referencia empleados por la propia Comisión, adaptando sus demandas a los nuevos enfoques institucionales puesto que aquellas estrategias formuladas en los términos que la Comisión quiere oír suelen tener mayor éxito. Otra estrategia por parte de los actores consiste en la búsqueda del punto de acceso a la agenda más favorable («venue shopping») ${ }^{36}$ : el consabido pluralismo de la UE hace que

terest groups \& Advocacy, 3, 2 (2014): 188-201.

${ }^{31}$ Erving Goffman, Frame analysis: los marcos de la experiencia (Madrid: Centro de Investigaciones Sociológicas, 2006).

${ }^{32}$ Vivien Schmidt, «Taking ideas and discourse seriously: explaining change through discursive institutionalism as the fourth "new institutionalism"», European Political Science Review, 2 , 1 (2010): 1-25.

33 «Skilled actors» en la terminología de Neil Fligstein y Doug McAdam, A theory of fields (Oxford: Oxford University Press, 2012): 52-55.

${ }^{34}$ SidneyTarrow. El poder en movimiento: los movimientos sociales, la acción colectiva y la política. (Madrid: Alianza editorial, 2004): 209-216.

35 Frida Boräng et al., «Identifying frames...

${ }^{36}$ Falk Daviter, «Policy framing in the European Union», Journal of European Public Policy 14. 4 (2007): 663-664; Sebastiann Princen, Agenda-setting in the European Union (Basingstoke: Palgrave Macmillan, 2009): 27-28. 
cada institución tenga su propia agenda y que por lo tanto una propuesta se pueda ver radicalmente reformulada en función del nivel al que se plantee. La mayor parte de políticas susceptibles de ser reguladas en el ámbito de la UE pueden afectar a varios servicios o direcciones generales de la Comisión o el Parlamento, pero el tipo de propuesta será radicalmente distinta en función de qué institución establezca el marco de referencia. En este contexto el encuadre y la definición del marco resultan fundamentales a la hora de definir el problema a tratar y de formular políticas públicas alternativas. En este sentido la reglamentación de los medios de comunicación en la UE será muy diferente en función de que se plantee como una cuestión que afecta fundamentalmente a las libertades públicas, a la política de competencia o a la innovación tecnológica y los derechos de autor ${ }^{37}$. A nivel estructural también cabe destacar la importancia de los encuadres en los cambios en la agenda, es decir el cambio del foco de una política de un objetivo a otro. Por ejemplo el cambio de objetivos de la PAC hacia la sostenibilidad y el medio ambiente y no a la producción tiene que ver con las metáforas sobre los lagos de leche y las montañas de mantequilla a los que habría dado lugar el productivismo de los años anteriores.

Por último se está desarrollando un nuevo enfoque, también de origen norteamericano, sobre las ideas en el proceso político que tiene que ver con los relatos de política pública ${ }^{38}$. Este enfoque argumenta que la política y la vida social están construidas mediante estructuras narrativas, y que por lo tanto también es necesario entender la elaboración de las políticas públicas como una competición por el establecimiento de relatos. Este enfoque también se aleja de las perspectivas más interpretativas al proponer un conjunto de hipótesis verificables, aunque la metodología de análisis de los relatos es menos clara, dando lugar a un debate sobre la coherencia epistemológica de la propuesta ${ }^{39}$. Además la perspectiva teórica se aleja de los argumentos sobre el «uso» de las ideas en la medida en que los defensores de la construcción narrativa de las políticas le prestan menor atención a la agencia en la construcción de los mismos y naturalizan la tendencia a actuar socialmente mediante relatos ${ }^{40}$. Si bien los enfoques sobre los relatos en la UE se han centrado hasta ahora fundamentalmente en la dimensión cultural de la in-

37 Alison J. Harcourt, «EU media ownership regulation: conflict over the definition of alternatives», JCMS: Journal of Common Market Studies, 36.3 (1998): 369-389.

38 Mark K. McBeth, Michael D. Jones y Elizabeth A. Shanahan, «The Narrative Policy Framework», en Paul Sabatier, y Christopher Weible (eds) Theories of the Policy Process (Boulder, CO: Westview Press, 2014).

39 Michael D. Jones, y Claudio M. Radaelli, «The Narrative Policy Framework: Child or Monster?», Critical Policy Studies, 9.3 (2015): 339-355.

40 Barbara Czarniawska, Narratives in social science research (Sage, 2004), p. 3. 
tegración europea o en la posición de los intelectuales ante la misma ${ }^{41}$ más que en las políticas públicas, el interés de las instituciones por los relatos y la ya mencionada similitud del proceso político estadounidense y el europeo permiten anticipar la próxima aplicación de este enfoque a la UE.

\section{Diferentes tipos de coaliciones}

El marco de las coaliciones promotoras, desarrollado en primer lugar en el contexto norteamericano, parece apropiado en un contexto multinivel caracterizado por la presencia de numerosos actores con veto y la necesidad de actuar en coalición en el largo plazo - el modelo enfatiza que los cambios en la agenda sólo son visibles en el plazo de una década - como es el de la Unión Europea, según ha reconocido su propio creador ${ }^{42}$. Lo significativo en el contexto de este artículo es que esta propuesta se organiza en torno a un modelo de círculos concéntricos de creencias más o menos intensas y por lo tanto adaptables. En este sentido las coaliciones promotoras reúnen a actores de naturaleza y posiciones diferentes y cuyas preferencias no están completamente alineadas pero que comparten una creencia nuclear. De este modo aunque puedan actuar en ámbitos y para fines distintos: lo que les reúne es una creencia compartida sobre la necesidad de la necesidad de abordar un problema o proponer una determinada política. Más allá de esta creencia nuclear y compartida existe un segundo y tercer círculo de creencias menos ancladas y por lo tanto más adaptables. Este argumento abre una puerta a entender que los miembros de la coalición pueden actuar con una cierta autonomía y adaptarse a las ventanas de oportunidad que surjan para promover propuestas limitadas que contribuyan a promover el argumento central. Al mismo tiempo permite establecer una relación con el establecimiento de marcos - las organizaciones pueden adaptar la presentación de su solución a diferentes interlocutores - y a la actuación estratégica, aceptando un cambio en sus preferencias y creencias secundarias para poder influir en la más importante.

Este modelo ha suscitado bastante interés para estudiar el desarrollo de cambios importantes en la agenda en torno a demandas con una cierta complejidad científica y que implican a actores diversos en ámbitos tales como la política de apoyo a las turbinas eólicas ${ }^{43} \mathrm{o}$ al sector de los hidrocarbu-

${ }^{41}$ Lacroix y Nicolaïdis European Stories...

${ }^{42}$ Paul Sabatier, «The advocacy coalition framework: revisions and relevance for Europe», Journal of European public policy, 5.1 (1998): 98-130.

43 Joseph Szarka, «Bringing interest back in: using coalition theories to explain European wind power policies», Journal of Europen Public Policy, 17.6 (2010): 836-53. 
$\operatorname{ros}^{44}$. Sin embargo el propio autor ha señalado que la adaptación de este enfoque a la Unión Europea requiere una serie de adaptaciones En este sentido Rozbicka señala que resulta difícil encontrar coaliciones estables en un plazo largo en un contexto tan cambiante y con tantas ventanas de oportunidad como el de la UE, puesto que la mayor parte de miembros de una coalición va a encontrar oportunidades para desviarse del objetivo colectivo en un determinado momento ${ }^{45}$. Por ejemplo en el año 2003 durante la Convención europea las organizaciones de inspiración religiosa y los representantes de las regiones se desvincularon de las ONGs que defendían el reconocimiento de la sociedad civil en el tratado y con las que llevaban trabajando conjuntamente desde finales de los 90 porque tuvieron la oportunidad de conseguir un reconocimiento diferenciado ${ }^{46}$.

Uno de los aspectos centrales del análisis de las coaliciones promotoras tiene que ver con el aprendizaje y el conocimiento. En este sentido el tipo de influencia para explicar el éxito de las coaliciones que propone este modelo se aproxima del de las comunidades epistémicas. Este modelo de análisis propuesto en los años 90 para explicar la capacidad de establecer la agenda de las numerosas organizaciones internacionales de carácter técnico ${ }^{47}$ ha tenido una aplicación casi natural al ámbito de la $\mathrm{UE}^{48}$. Esto se debe al carácter «tecnocrático» de muchas de las ventanas de oportunidad que ofrece el proceso político de la UE a los diferentes actores, tales como los grupos de expertos de la Comisión, los comités de comitología o las consultas previas preparatorias de evaluaciones de impacto, por no hablar de la creciente «agencificación» de numerosos procesos.

Princen define estas comunidades como grupos más o menos permanentes o al menos estables de expertos que «leen las mismas publicaciones, van a las mismas conferencias y reuniones, y de esta manera comparten un entendimiento de los problemas de política pública y de las opciones políticas disponibles para abordarlos» ${ }^{49}$. Este tipo de grupos son más frecuentes en las áreas de una cierta complejidad técnica - como por ejemplo la salud

${ }^{44}$ Rainer Eising, «The access of business interests to EU institutions: towards elite pluralism?», Journal of European Public Policy, 14.3 (2007): 384-403.

45 Patricja Rozbicka, «Advocacy coalitions: influencing the policy process in the EU», Journal of European Public Policy, 20.6 (2013): 850-851.

46 Luis Bouza García, Participatory democracy and..., 102-104.

47 Peter M. Haas, «Introduction: epistemic communities and international policy coordination», International organization, 46.1 (1992): 1-35.

48 Anthony R. Zito, «Epistemic communities, collective entrepreneurship and European integration», Journal of European public policy, 8.4 (2001): 585-603.

49 Sebastiaan Princen, Agenda-setting in the European Union (Basingstoke: Palgrave Macmillan, 2009) 151-152, traducción del autor. 
pública o el cambio climático ${ }^{50}$ - en las cuales el grado de conocimiento científico es un argumento fundamental a la hora de promover unas soluciones u otras, si bien es cierto que este es cada vez más el caso con el creciente uso de las regulaciones de impacto en el marco del proyecto «regular mejor ${ }^{51}$. Estas comunidades resultan importantes por dos motivos. Por una parte su naturaleza científica sugiere la sinceridad del convencimiento de sus miembros sobre la evidencia de cuál es la mejor propuesta. En este sentido aunque operen en el largo plazo - puesto que los marcos de los actores se adaptan lentamente a la nueva evidencia científica - potencialmente tienen un efecto más profundo puesto que actúan sobre las preferencias de los actores y no sólo en los aspectos superficiales del marco, por continuar la metáfora de Sabatier sobre los círculos concéntricos. Pero por otra parte tienen una capacidad de convencimiento especialmente alta debido a su composición: estas comunidades reúnen a expertos que comparten una temática de interés pero están ubicados en organizaciones distintas. Por lo tanto los miembros de la comunidad se convierten en promotores de causa dentro de sus organizaciones, facilitando la hegemonía de una propuesta una vez esta alcanza su grado de maduración.

\section{Algunas implicaciones metodológicas}

Uno de los aspectos problemáticos ${ }^{52}$ respecto a las coaliciones promotoras o las redes de conocimiento tiene que ver con la dificultad de proporcionar evidencia sobre los cambios de preferencias, o ni siquiera de pasar del estado de metáfora ${ }^{53}$ a la hora de evidenciar la existencia y la importancia de redes de políticas públicas. Sin embargo argumentamos que la perspectiva epistemológica y teórica sobre los usos del conocimiento así como algunas innovaciones metodológicas relativas al uso de mejores técnicas de análisis de textos y de redes tienen un fuerte potencial a la hora de transformar dichas «metáforas» en hipótesis verificables.

El análisis de los marcos de referencia y los aspectos teóricos sobre la «importancia de las ideas» reenvían a evaluaciones cualitativas que pueden ser difíciles de verificar empíricamente y sobre todo de generalizar. Además

50 Ibid.

51 Comisión Europea, Communication from the Commission. «Action Plan «Simplifying and improving the regulatory environment, COM/2002 278 (2002).

52 Hussein Kassim, «Policy networks, networks and European Union policy making: A sceptical view», West European Politics 17.4 (1994): 15-27.

53 Keith Dowding, «Model or metaphor? A critical review of the policy network approach», Political studies, 43.1 (1995): 136-158. 
estas perspectivas se alejan de buen número de los enfoques más frecuentes en torno a la elaboración de políticas públicas. Por lo tanto la comprensión del conocimiento y las ideas como un marco y al tiempo como un recurso puede servir para derivar hipótesis verificables sobre la correspondencia entre marcos de interpretación - que pueden estudiarse mediante análisis de textos y entrevistas con los actores - y formas de acción colectiva que pueden objetivarse mediante la presencia de dichos actores en consultas con la Comisión, encuestas o análisis de redes. Este tipo de procedimiento puede permitir verificar hipótesis sobre la importancia de los marcos para la acción colectiva o para el cambio de políticas públicas (es decir, ¿cambian las formas de actuación y el contenido de las propuestas tras cambios en los marcos de los actores? Esta perspectiva invita por lo tanto al empleo de métodos mixtos, lo cual resulta especialmente interesante gracias al reciente interés en los estudios sobre grupos de interés de métodos cuantitativos de análisis de texto - con qué frecuencia recogen los textos oficiales los argumentos de los grupos ${ }^{54}-\mathrm{y}$ las propuestas para sistematizar los marcos de referencia ${ }^{55}$.

\section{Conclusión}

El repaso de la literatura sobre el papel de los actores en el establecimiento de la agenda en la UE confirma la pujanza de los enfoques centrados en el conocimiento y las ideas de forma sostenida a lo largo de casi dos décadas. Se trata además de un enfoque caracterizado por una gran diversidad interna y debates relativamente enconados dadas las diferencias epistemológicas y teóricas. En todo caso estos enfoques tienen la característica común de alejarse de las explicaciones centradas en las instituciones comunitarias y de la teoría de la decisión racional para centrarse en la importancia de las ideas y en los actores. Además de por poner en cuestión los enfoques teóricos predominantes el éxito de este enfoque resulta llamativo debido a que al menos en un primer momento la inexistencia de una opinión pública y de una esfera pública unificada debería ser un factor que dificultase estrategias orientadas al debate y la persuasión en favor de explicaciones basadas en la capacidad de los actores de alcanzar transacciones.

El artículo señala sin embargo que este éxito resulta menos sorprendente si se tiene en cuenta la naturaleza de un proceso político multinivel caracterizado por el pluralismo de los actores, la complejidad técnica de

\footnotetext{
${ }^{54}$ Klüver Lobbying in...

55 Boräng et al. «Identifying frames...
} 
los asuntos y la existencia de numerosos puntos de veto. En este contexto la introducción de cambios en la agenda requiere de la suma de actores que puedan superar los equilibrios inducidos. En este contexto la información y las ideas resultan importantes en un plano instrumental como sincero. De manera instrumental la información es un recurso clave en el proceso político comunitario y la adaptación de los marcos de referencia puede servir para ampliar coaliciones o para cambiar el foro de la elaboración de las políticas públicas hacia el que pueda ser más favorable a la coalición o actor en cuestión. En términos sinceros la importancia de la reputación y la complejidad de los temas, así como la constante búsqueda de legitimidad por parte de las instituciones comunitarias contribuyen a que se puedan construir ideas o causas casi imposibles de resistir por parte de potenciales detractores.

La aparente contradicción entre la consideración sincera e instrumental de la capacidad de persuasión de los actores comunitarios se puede reconciliar a través de una perspectiva sociológica sobre los «usos de Europa» por parte de sus actores. Esta es una perspectiva que asume que los actores persiguen sus objetivos y estrategias de forma consecuente en un entorno de racionalidad limitada por sus creencias y valores, al igual que los del entorno. De este modo tienen un margen de maniobra limitado a la hora de adaptar sus propuestas al contexto que pueda resultar más favorable. El grado de aceptación de los enfoques centrados en los marcos a pesar de las diferencias entre cada uno de los enfoques considerados en el artículo confirma la existencia de dinámicas de acción colectiva en torno a las ideas en el segmentado espacio de debate y elaboración de políticas públicas de la UE.

Sin embargo conviene ser cauto sobre el potencial explicativo de estos enfoques precisamente por su la riqueza interna y diversidad así como por sus diferentes grados de adaptación a la UE. En primer lugar por enriquecedora que sea, la tendencia a la fragmentación dificulta la comparación entre enfoques, y aún más la adopción de metodologías que permitan la verificación sistemática de las hipótesis avanzadas, si bien recientemente se están desarrollando enfoques de métodos mixtos centrados en el análisis cuantitativo de textos y marcos ${ }^{56}$. En segundo lugar todos los estudios citados expresan el potencial del enfoque utilizado en cada uno al tiempo que formulan cautelas importantes sobre la posibilidad de su aplicación sistemática a la UE. De este modo los autores señalan la capacidad explicativa de las comunidades epistémicas sobre algunas decisiones técnicamente complejas o de la excepcionalidad de coaliciones promotoras de largo alcance. Esto se

56 Klüver Lobbying in... Boräng et al. «Identifying frames» 
debe sin duda al hecho de que numerosos de estos enfoques se originan en el proceso político estadounidense, que comparte numerosas características con el de la UE pero del que le separan aspectos importantes en el plano institucional como en el de las ideas dada la capacidad de aquellos actores de recurrir con más facilidad a la opinión pública.

\section{Bibliografía}

Aldrin, Philippe, «Producteurs, courtiers et experts de l'information européenne», en D. Georgakakis, Le champ de l'eurocratie, Economica (2012): 151-181

Baumgartner Frank R., Bryan D. Jones y Peter B. y Mortensen. «Punctuated equilibrium theory: Explaining stability and change in public policymaking», en Sabatier Paul A. y Christopher Weible (eds), Theories of the policy process, (2014): 59-103.

Boräng, Frida, Rainer Eising, Heike Klüver, Christine Mahoney, Daniel Naurin, Daniel Rasch y Patricja Rozbicka. «Identifying frames: A comparison of research methods», Interest groups \& Advocacy, 3.2 (2014): 188-201.

Bouwen, Peter y Margaret McCown, «Lobbying versus litigation: political and legal strategies of interest representation in the European Union», Journal of European Public Policy, 14 (2007): 422-43.

Bouza García, Luis, Participatory Democracy and Civil Society in the EU: AgendaSetting and Institutionalisation, 2015.

Chalmers, Damian, «The reconstitution of European public spheres», European Law Journal, 9.2, (2003): 127-189.

Chalmers, Adam W., «Interests, influence and information: comparing the influence of interest groups in the European Union», Journal of European Integration 33 (2011): 471-86.

Chopin, Thierry, «Towards a Genuine European Executive Power: from Governance to Government», European Issues, 274 (2013) http://www.robert-schuman. eu/doc/questions_europe/qe-274-en.pdf

Comisión Europea, Communication from the Commission - Action Plan Simplifying and improving the regulatory environment, $\mathrm{COM} / 2002$ 278, 2002.

Czarniawska, Barbara, Narratives in social science research. Sage, 2004.

Dahl, Robert A. «A Critique of the Ruling Elite Model», American Political Science Review, 52.2 (1958): 463-469

Daviter, Falk, «Policy framing in the European Union», Journal of European Public Policy, 14.4 (2007): 654-666.

Diez Medrano, Juan y Emily Gray, «Framing the European Union in National Public Spheres», en Koopmans, Ruud y Paul Statham (eds), The making of a European public sphere: Media discourse and political contention, Cambridge University Press (2010): 125-150.

Dowding, Keith, «Model or metaphor? A critical review of the policy network approach», Political studies, 43.1 (1995): 136-158. 
Dür, Andreas, Peter Bernhagen y David Marshall, «Interest Group Success in the European Union When (and Why) Does Business Lose?», Comparative Political Studies, 48(8) (2015): 951-983.

Eising, Rainer, «The access of business interests to EU institutions: towards elite pluralism?», Journal of European Public Policy, 14.3 (2007): 384-403.

Engel, Filip, «Analyzing Policy Learning in European Union Policy Formulation: The Advocacy Coalition Framework Meets New-Institutional Theory», Bruges Political Research Papers, 5 (2007).

Eriksen, Erik Odvar, «Conceptualising European public spheres. General, segmented and strong publics», en J. E. Fossum y P. Schlesinger, eds. The European Union and the Public Sphere. A communicative space in the making?, London: Routledge, 2007.

Fligstein, Neil y Doug McAdam, A theory of fields, Oxford, Oxford University Press, 2012.

Follesdal, Andreas y Simon Hix, «Why There is a Democratic Deficit in the EU: A Response to Majone and Moravcsik», Journal of Common Market Studies 44.3 (2006): 533-562.

Goffman, Erving, Frame analysis: los marcos de la experiencia, Madrid: Centro de Investigaciones Sociológicas, 2006.

Haas, Peter M. «Introduction: epistemic communities and international policy coordination», International organization, 46.1 (1992): 1-35.

Harcourt, Alison J. «EU media ownership regulation: conflict over the definition of alternatives», JCMS: Journal of Common Market Studies, 36.3 (1998): 369-389.

Jacquot, S,ophie y Cornelia Woll, «Action publique Européenne: les acteurs stratégiques face a l'Europe», Politique européenne, 2 (2008): 161-192.

Jones, Michael D., y Claudio M. Radaelli, C. M. «The Narrative Policy Framework: Child or Monster?», Critical Policy Studies, 9.3 (2015): 339-355.

Kassim, Hussein «Policy networks, networks and European Union policy making: A sceptical view», West European Politics 17.4 (1994): 15-27.

Kingdon John W. Agendas, Alternatives, and Public Policies. New York: Longman, 2003.

Klüver, Heike, Lobbying in the European Union: interest groups, lobbying coalitions, and policy change. Oxford: OUP Oxford, 2013.

Lacroix, Justine y Nicolaïdis Kalypso European Stories: Intellectual Debates on Europe in National Contexts. Oxford, 2010.

Lindberg, Leon y Stuart Scheingold Europe's Would Be Polity. Prentice Hall, Englewood Cliffs, 1970.

Lindblom, Charles E. Politics and markets: the world's political-economic systems, New York: Basic: 1977.

Mahoney, Christine, «Networking Vs. Allying: The Decision of Interest Groups to Join Coalitions in the US and the EU», Journal of European Public Policy, 14.3 (2007): 363-383.

Mahoney, Christine, «The Role of Interest Groups in Fostering Citizen Engagement: The Determinants of Outside Lobbying», en Maloney, W. y van Deth, J. Civil Society and Governance in Europe. From National to International Linkages, Cheltenham: Edward Elgar, 2008. 
McBeth Mark K., Michael D. Jones y Elizabet A. Shanahan, E.A. «The Narrative Policy Framework» en Sabatier, Paul A. y Christopher Weible (eds). Theories of the Policy Process, Boulder, CO: Westview Press, 2014.

Muller, Pierre, «L'analyse cognitive des politiques publiques: vers une sociologie politique de l'action publique», Revue française de science politique (2000): 189-207.

Princen, Sebastiaan, Agenda-setting in the European Union. Basingstoke: Palgrave Macmillan, 2009.

Princen, Sesbastiaan, «Punctuated equilibrium theory and the European Union», Journal of European Public Policy, 20.6 (2013): 854-870.

Quittkat, Christine, «The European Commission's online consultations: a success story?», JCMS: Journal of Common Market Studies 49.3 (2011): 653-674.

Rozbicka, Patrycja, «Advocacy coalitions: influencing the policy process in the EU», Journal of European Public Policy, 20.6 (2013): 838-853.

Sabatier, P.A., «The advocacy coalition framework: revisions and relevance for Europe», Journal of European public policy, 5.1 (1998): 98-130.

Saurugger, Sabine (dir), Les approches sociologiques de l'intégration européenne: Perspectives critiques. Politique européenne ,25 (2008): 5-230.

Schmidt, Vivien, «Taking ideas and discourse seriously: explaining change through discursive institutionalism as the fourth "new insitutionalism"», European Political Science Review, 2.1 (2010): 1-25.

Smismans, Stijn, «European civil society: Shaped by discourses and institutional interests», European law journal, 9.4 (2003): 473-495.

Szarka, Joseph, «Bringing interest back in: using coalition theories to explain European winf power policies», Journal of Europen Public Policy 17.6 (2010): 83653.

Tarrow, Sidney, El poder en movimiento: los movimientos sociales, la acción colectiva y la política. Madrid: Alianza editorial, 2004.

Tsebelis George y Yataganas, Xenophon, «Veto Players and Decision-making in the EU After Nice», Journal of Common Market Studies, 40.2 (2002): 283-307.

Zito, Anthony R., «Epistemic Communities, Collective Entrepreneurship and European Integration», Journal of European Public Policy, 8.4 (2001): 585-603. 


\section{Derechos de autor (Copyright)}

Los derechos de autor (distribución, comunicación pública, reproducción e inclusión en bases de datos de indexación y repositorios institucionales) de esta publicación pertenecen a la editorial Universidad de Deusto. El acceso al contenido digital de cualquier número de Cuadernos Europeos de Deusto (CED) es gratuito, transcurridos 6 meses desde su publicación. Los trabajos podrán descargarse, copiar y difundir, sin fines comerciales y según lo previsto por la ley. Así mismo, los trabajos editados en CED pueden ser publicados con posterioridad en otros medios o revistas, siempre que el autor indique con claridad y en la primera nota a pie de página que el trabajo se publicó por primera vez en CED, con indicación del número, año, páginas y DOI (si procede). 